\title{
On the Study of Global Solutions for a Nonlinear Equation
}

\author{
Haibo Yan ${ }^{1,2}$ and Ls Yong ${ }^{1}$ \\ ${ }^{1}$ Department of Applied Mathematics, Southwestern University of Finance and Economics, Chengdu 610074, China \\ ${ }^{2}$ Department of Mathematics, Xinjiang University of Finance and Economics, Urumqi 830012, China
}

Correspondence should be addressed to Haibo Yan; wengxiang00@163.com

Received 3 February 2014; Accepted 27 March 2014; Published 14 April 2014

Academic Editor: Yonghong Wu

Copyright (C) 2014 H. Yan and L. Yong. This is an open access article distributed under the Creative Commons Attribution License, which permits unrestricted use, distribution, and reproduction in any medium, provided the original work is properly cited.

\begin{abstract}
The well-posedness of global strong solutions for a nonlinear partial differential equation including the Novikov equation is established provided that its initial value $v_{0}(x)$ satisfies a sign condition and $v_{0}(x) \in H^{s}(R)$ with $s>3 / 2$. If the initial value $v_{0}(x) \in H^{s}(R)(1 \leq s \leq 3 / 2)$ and the mean function of $\left(1-\partial_{x}^{2}\right) v_{0}(x)$ satisfies the sign condition, it is proved that there exists at least one global weak solution to the equation in the space $v(t, x) \in L^{2}\left([0,+\infty), H^{s}(R)\right)$ in the sense of distribution and $v_{x} \in L^{\infty}([0,+\infty) \times R)$.
\end{abstract}

\section{Introduction}

Recently, $\mathrm{Wu}$ [1] obtained the existence of local solutions in the space $C\left([0, T) ; H^{s}(R)\right) \cap C^{1}\left([0, T) ; H^{s-1}(R)\right)$ with $s>3 / 2$ for the following nonlinear equation:

$$
\begin{aligned}
& v_{t}-v_{t x x}+k v^{m} v_{x}+(m+3) v^{m+1} v_{x} \\
& =(m+2) v^{m} v_{x} v_{x x}+v^{m+1} v_{x x x}+\lambda\left(v-v_{x x}\right),
\end{aligned}
$$

where $m \geq 0$ is a natural number, $k \geq 0$, and $\lambda$ is a constant. Letting $m=0$ and $\lambda=0$, (1) becomes the Camassa-Holm equation [2]. If $m=1, k=0$, and $\lambda=0$, (1) reduces to the Novikov equation [3].

A lot of works have been carried out to study various dynamic properties for the Camassa-Holm and the Novikov equations. Xin and Zhang [4] proved that there exists a global weak solution for the Camassa-Holm equation in the space $H^{1}(R)$ without the assumption of sign conditions on the initial value. Coclite et al. [5] investigated the global weak solutions for a generalized hyperelastic rod wave equation or a generalized Camassa-Holm equation. It is shown in Constantin and Escher [6] that the blowup occurs in the form of breaking waves; namely, the solution remains bounded but its slope becomes unbounded in finite time. After wave breaking, the solution can be continued uniquely either as a global conservative weak solution [7] or a global dissipative solution [8-10]. The periodic and the nonperiodic
Cauchy problems for the Novikov equation were discussed by Grayshan [11] in the Sobolev space. Using the Galerkintype approximation method, Himonas and Holliman [12] established the well-posedness for the Novikov model in the Sobolev space $H^{s}(R)$ with $s>3 / 2$ on both the line and the circle. The scattering theory was employed in Hone et al. [13] to find nonsmooth explicit soliton solutions with multiple peaks for the Novikov equation. Wu and Zhong [14] proved the existence of local strong and weak solutions for a generalized Novikov equation.

The objective of this work is to study (1) with $k=0$. Namely, we investigate the problem

$$
\begin{gathered}
v_{t}-v_{t x x}+(m+3) v^{m+1} v_{x} \\
=(m+2) v^{m} v_{x} v_{x x}+v^{m+1} v_{x x x}+\lambda\left(v-v_{x x}\right), \\
v(0, x)=v_{0}(x),
\end{gathered}
$$

where $m, k$, and $\lambda$ are described in (1). Assuming that the initial value $v_{0}(x)$ satisfies a sign condition and $v_{0}(x) \epsilon$ $H^{s}(R), s>3 / 2$, we will show that there exists a unique global strong solution in the Sobolev space $C\left([0, \infty) ; H^{s}(R)\right) \cap$ $C^{1}\left([0, \infty) ; H^{s-1}(R)\right)$. If the initial value $v_{0}(x) \in H^{s}(R)(1 \leq$ $s \leq 3 / 2)$ and the mean function of $\left(1-\partial_{x}^{2}\right) v_{0}(x)$ satisfies the sign condition, it is shown that there exists at least one global weak solution to the equation in the space $v(t, x) \epsilon$ 
$L^{2}\left([0,+\infty), H^{s}(R)\right)$ in the sense of distribution and $v_{x} \in L^{\infty}$ $([0,+\infty) \times R)$.

The structure of this paper is as follows. The main results are given in Section 2. Several lemmas are given in Section 3. Section 4 establishes the proof of the main results.

\section{Main Results}

We define

$$
\phi(x)= \begin{cases}e^{1 /\left(x^{2}-1\right)}, & |x|<1 \\ 0, & |x| \geq 1\end{cases}
$$

and let $\phi_{\varepsilon}(x)=\varepsilon^{-1 / 4} \phi\left(\varepsilon^{-1 / 4} x\right)$ with $0<\varepsilon<1 / 4$. For the convolution $v_{\varepsilon 0}=\phi_{\varepsilon} \star v_{0}$, we know that $v_{\varepsilon 0} \in C^{\infty}$ for any $v_{0} \in H^{s}$ with $s>0$. Notation $\left(1-\partial_{x}^{2}\right) v \in N^{+}(R)$ (or equivalently (1$\left.\left.\partial_{x}^{2}\right) v \in N^{-}(R)\right)$ means that the mean function of $\left(1-\partial_{x}^{2}\right) v$ is nonnegative; namely, $\left(1-\partial_{x}^{2}\right) v \star \phi_{\varepsilon} \geq 0$ (or equivalently ( $1-$ $\left.\partial_{x}^{2}\right) v \star \phi_{\varepsilon} \leq 0$ ) for an arbitrary sufficiently small $\varepsilon>0$. For $T>$ 0 and nonnegative number $s$, we let $C\left([0, T) ; H^{s}(R)\right)$ denote the Frechet space of all continuous $H^{s}$-valued functions on $[0, T)$ and write $\Lambda=\left(1-\partial_{x}^{2}\right)^{1 / 2}$. (2).

We state the result of global strong solutions for problem

Theorem 1. Let $v_{0}(x) \in H^{s}(R), s>3 / 2$, and $\left(1-\partial_{x}^{2}\right) v_{0} \geq 0$ for all $x \in R$ or $\left(1-\partial_{x}^{2}\right) v_{0} \leq 0$ for all $x \in R$. Then problem (2) has a unique strong solution satisfying

$$
v(t, x) \in C\left([0, \infty) ; H^{s}(R)\right) \cap C^{1}\left([0, \infty) ; H^{s-1}(R)\right) .
$$

Definition 2. A function $v(t, x) \in L^{2}\left([0,+\infty), H^{s}(R)\right)$ is called a global weak solution to problem (2) if for every $T>0$ and all $\varphi(t, x) \in C_{0}^{\infty}([0, T] \times R)$, it holds that

$$
\begin{gathered}
\int_{0}^{T} \int_{R}\left[v_{t}-v_{t x x}+(m+3) v^{m+1} v_{x}-(m+2) v^{m} v_{x} v_{x x}\right. \\
\left.-v^{m+1} v_{x x x}-\lambda\left(v-v_{x x}\right)\right] \varphi(t, x) d x d t=0
\end{gathered}
$$

with $v(0, x)=v_{0}(x)$.

Now we give the main result of global weak solution for problem (2).

Theorem 3. Let $v_{0}(x) \in H^{s}(R), 1 \leq s \leq 3 / 2,\left(1-\partial_{x}^{2}\right) v_{0} \in$ $N^{+}(R)$ (or equivalently $\left(1-\partial_{x}^{2}\right) v_{0} \in N^{-}(R)$ ). Then problem (2) has a unique global weak solution $v(t, x) \in L^{2}\left([0,+\infty), H^{s}(R)\right)$ in the sense of distribution and $v_{x} \in L^{\infty}([0,+\infty) \times R)$.

\section{Several Lemmas}

Lemma 4 (see [1]). Let $v_{0}(x) \in H^{s}(R)$ with $s>3 / 2$. Then the Cauchy problem (2) has a unique local solution

$$
v(t, x) \in C\left([0, T) ; H^{s}(R)\right) \cap C^{1}\left([0, T) ; H^{s-1}(R)\right),
$$

where $T>0$ depends on $\left\|v_{0}\right\|_{H^{s}(R)}$.
Using the first equation of system (2) derives

$$
\frac{d}{d t} \int_{R}\left(v^{2}+v_{x}^{2}\right) d x=2 \lambda \int_{R}\left(v^{2}+v_{x}^{2}\right) d x,
$$

which yields the conservation law

$$
\begin{aligned}
\int_{R}\left(v^{2}+v_{x}^{2}\right) d x= & \int_{R}\left(v_{0}^{2}+v_{0 x}^{2}\right) d x \\
& +2 \lambda \int_{0}^{t} \int_{R}\left(v^{2}+v_{x}^{2}\right) d x d t .
\end{aligned}
$$

Lemma 5 (see [1]). Let $s>3 / 2$ and the function $v(t, x)$ is a solution of problem (2) and the initial data $v_{0}(x) \in H^{s}$. Then the following inequalities hold:

$$
\begin{gathered}
\|v\|_{H^{1}}^{2} \leq \int_{R}\left(v^{2}+v_{x}^{2}\right) d x \leq \int_{R}\left(v_{0}^{2}+v_{0 x}^{2}\right) d x, \quad \text { if } \lambda \leq 0 . \\
\|v\|_{H^{1}}^{2} \leq \int_{R}\left(v^{2}+v_{x}^{2}\right) d x \leq e^{2 \lambda t} \int_{R}\left(v_{0}^{2}+v_{0 x}^{2}\right) d x, \quad \text { if } \lambda>0 .
\end{gathered}
$$

For $q \in(0, s-1]$, there is a constant $c$ such that

$$
\begin{aligned}
& \int_{R}\left(\Lambda^{q+1} v\right)^{2} d x \\
& \leq \int_{R}\left(\Lambda^{q+1} v_{0}\right)^{2} d x \\
& \quad+c \int_{0}^{t}\|v\|_{H^{q+1}}^{2}\left(|\lambda|+\left(\|v\|_{L^{\infty}}^{m-1}+\|v\|_{L^{\infty}}^{m}\right)\left\|v_{x}\right\|_{L^{\infty}}\right. \\
& \left.+\|v\|_{L^{\infty}}^{m-1}\left\|v_{x}\right\|_{L^{\infty}}^{2}\right) d \tau .
\end{aligned}
$$

For $q \in[0, s-1]$, there is a constant $c$ such that

$$
\begin{aligned}
\left\|v_{t}\right\|_{H^{q}} \leq c\|v\|_{H^{q+1}}\left(|\lambda|+\left(\|v\|_{L^{\infty}}^{m-1}+\|v\|_{L^{\infty}}^{m}\right)\|v\|_{H^{1}}\right. \\
\left.+\|v\|_{L^{\infty}}^{m}\left\|v_{x}\right\|_{L^{\infty}}+\|v\|_{L^{\infty}}^{m-1}\left\|v_{x}\right\|_{L^{\infty}}^{2}\right) .
\end{aligned}
$$

Consider the differential equation

$$
\begin{gathered}
p_{t}=v^{m+1}(t, p), \quad t \in[0, T), \\
p(0, x)=x,
\end{gathered}
$$

where $v(t, x)$ is the solution of problem (2) and $T$ is the maximal existence time of the solution.

Lemma 6. Let $v_{0} \in H^{s}(R), s \geq 3$, and let $T>0$ be the maximal existence time of the solution to problem (2). Then system (12) has a unique solution $p(t, x) \in C^{1}([0, T) \times R)$. Moreover, the map $p(t, \cdot)$ is an increasing diffeomorphism of $R$ with $p_{x}(t, x)>0$ for $(t, x) \in[0, T) \times R$.

Proof. From Lemma 4, we know that there exists a unique solution

$$
v(t, x) \in C\left([0, T) ; H^{s}(R)\right) \cap C^{1}\left([0, T) ; H^{s-1}(R)\right) .
$$


The Sobolev imbedding theorem derives $H^{s}(R) \in C^{1}(R)$. This means that two functions $v(t, x)$ and $v_{x}(t, x)$ are bounded, Lipschitz in space and $C^{1}$ in time. Using the existence and uniqueness theorem of ordinary differential equations, we derive that problem (12) has a unique solution $p(t, x) \in C^{1}$ $([0, T) \times R)$.

Differentiating (12) with respect to $x$ gives rise to

$$
\begin{gathered}
\frac{d}{d t} p_{x}=(m+1) v^{m} v_{x}(t, p) p_{x}, \quad t \in[0, T), \\
p_{x}(0, x)=1,
\end{gathered}
$$

from which we obtain

$$
p_{x}(t, x)=\exp \left(\int_{0}^{t}(m+1) v^{m} v_{x}(\tau, p(\tau, x)) d \tau\right) .
$$

For every $T^{\prime}<T$, applying the Sobolev imbedding theorem results in

$$
\sup _{(\tau, x) \in\left[0, T^{\prime}\right) \times R}\left|v_{x}(\tau, x)\right|<\infty .
$$

Therefore, we know that there exists a constant $M>0$ such that $p_{x}(t, x) \geq e^{-M t}$ for $(t, x) \in[0, T) \times R$. The proof is completed.

Lemma 7. Let $v_{0} \in H^{s}$ with $s \geq 3$, and let $T>0$ be the maximal existence time of the problem (2); it holds that

$$
y(t, p(t, x)) p_{x}^{2}(t, x)=y_{0}(x) e^{\int_{0}^{t}\left(m v^{m} v_{x}+\lambda\right) d \tau},
$$

where $(t, x) \in[0, T) \times R$ and $y:=v-v_{x x}$.

Proof. We have

$$
\begin{aligned}
\frac{d}{d t}[y( & \left.t, p(t, x)) p_{x}^{2}(t, x)\right] \\
= & y_{t} p_{x}^{2}+2 y p_{x} p_{x t}+y_{x} p_{t} p_{x}^{2} \\
= & y_{t} p_{x}^{2}+2 y(m+1) v^{m} v_{x} p_{x}^{2}+v^{m+1} y_{x} p_{x}^{2} \\
= & {\left[y_{t}+(m+2) v^{m} v_{x} y+y_{x} v^{m+1}\right] p_{x}^{2}+m v^{m} v_{x} y p_{x}^{2} } \\
= & {\left[v_{t}-v_{t x x}+(m+2) v^{m} v_{x}\left(v-v_{x x}\right)\right.} \\
& \left.+v^{m+1}\left(v_{x}-v_{x x x}\right)-\lambda\left(v-v_{x x}\right)\right] p_{x}^{2} \\
& +\left(m v^{m} v_{x}+\lambda\right) y p_{x}^{2} \\
= & {\left[v_{t}-v_{t x x}+(m+3) v^{m+1} v_{x}-(m+2) v^{m} v_{x} v_{x x}\right.} \\
& \left.-v^{m+1} v_{x x x}-\lambda\left(v-v_{x x}\right)\right] p_{x}^{2} \\
& +\left(m v^{m} v_{x}+\lambda\right) y p_{x}^{2} \\
= & \left(m v^{m} v_{x}+\lambda\right) y p_{x}^{2},
\end{aligned}
$$

from which we have

$$
y(t, p(t, x)) p_{x}^{2}(t, x)=p_{x}(0, x) y_{0}(x) e^{\int_{0}^{t}\left(m v^{m} v_{x}+\lambda\right) d \tau} .
$$

Using $p_{x}(0, x)=1$ completes the proof.
Lemma 8. If $v_{0} \in H^{s}(R), s \geq 3 / 2,\left(1-\partial_{x}^{2}\right) v_{0} \geq 0$ or $(1-$ $\left.\partial_{x}^{2}\right) v_{0} \leq 0$, then the solution of problem (2) satisfies

$$
\left\|v_{x}\right\|_{L^{\infty}} \leq\|v\|_{L^{\infty}} .
$$

Proof. We only need to prove this lemma for the case $v_{0}-$ $v_{0 x x} \geq 0$ since the proof of the other case $\left(1-\partial_{x}^{2}\right) v_{0} \leq 0$ is similar. It follows from Lemmas 6 and 7 that $v-v_{x x} \geq 0$. Letting $\xi(t, x)=v-v_{x x}$, we have

$$
v=\frac{1}{2} e^{-x} \int_{-\infty}^{x} e^{\eta} \xi(t, \eta) d \eta+\frac{1}{2} e^{x} \int_{x}^{\infty} e^{-\eta} \xi(t, \eta) d \eta,
$$

which derives

$$
\begin{aligned}
\partial_{x} v(t, x)= & -\frac{1}{2}\left(e^{-x} \int_{-\infty}^{x} e^{\eta} \xi(t, \eta) d \eta+e^{x} \int_{x}^{\infty} e^{-\eta} \xi(t, \eta) d \eta\right) \\
& +e^{x} \int_{x}^{\infty} e^{-\eta} \xi(t, \eta) d \eta \\
= & -v(t, x)+e^{x} \int_{x}^{\infty} e^{-\eta} \xi(t, \eta) d \eta \\
\geq & -v(t, x) .
\end{aligned}
$$

On the other hand, we have

$$
\begin{aligned}
\partial_{x} v(t, x)= & \frac{1}{2}\left(e^{-x} \int_{-\infty}^{x} e^{\eta} \xi(t, \eta) d \eta+e^{x} \int_{x}^{\infty} e^{-\eta} \xi(t, \eta) d \eta\right) \\
& -e^{-x} \int_{-\infty}^{x} e^{\eta} \xi(t, \eta) d \eta \\
= & v(t, x)-e^{-x} \int_{-\infty}^{x} e^{\eta} \xi(t, \eta) d \eta \\
\leq & v(t, x) .
\end{aligned}
$$

The inequalities (22) and (23) derive that inequality (20) is valid.

Lemma 9. For $s>0, u \in H^{s}(R)$, and $u_{\varepsilon}=\phi_{\varepsilon} \star u$, it holds that

$$
\begin{gathered}
\left\|u_{\varepsilon x}\right\|_{L^{\infty}} \leq c\left\|u_{x}\right\|_{L^{\infty}}, \\
\left\|u_{\varepsilon}\right\|_{H^{q}} \leq c, \quad \text { if } q \leq s, \\
\left\|u_{\varepsilon}\right\|_{H^{q}} \leq c \varepsilon^{(s-q) / 4}, \quad \text { if } q>s, \\
\left\|u_{\varepsilon}-u\right\|_{H^{q}} \leq c \varepsilon^{(s-q) / 4}, \quad \text { if } q \leq s, \\
\left\|u_{\varepsilon}-u\right\|_{H^{s}}=o(1),
\end{gathered}
$$

where $c$ is a constant independent of $\varepsilon$. 
The proof of this lemma can be found in $[15,16]$.

From Lemma 4, it derives that the Cauchy problem

$$
\begin{aligned}
& v_{t}-v_{t x x}=-(m+3) v^{m+1} v_{x}+(m+2) v^{m} v_{x} v_{x x} \\
&+v^{m+1} v_{x x x}+\lambda\left(v-v_{x x}\right) \\
&=-\frac{m+3}{m+2}\left(v^{m+2}\right)_{x}+\frac{1}{m+2} \partial_{x}^{3}\left(v^{m+2}\right) \\
&-(m+1) \partial_{x}\left(v^{m} v_{x}^{2}\right)+v^{m} v_{x} v_{x x}+\lambda\left(v-v_{x x}\right), \\
& v(0, x)=v_{\varepsilon 0}(x),
\end{aligned}
$$

has a unique solution $v$ depending on the parameter $\varepsilon$. We write $v_{\varepsilon}(t, x)$ to represent the solution of problem (25). Using Lemma 4 derives that $v_{\varepsilon}(t, x) \in C^{\infty}\left([0, T), H^{\infty}(R)\right)$ since $v_{\varepsilon 0}(x) \in C_{0}^{\infty}(R)$.

Lemma 10. Provided that $v_{0} \in H^{s}(R), 1 \leq s \leq 3 / 2$, and $\left(1-\partial_{x}^{2}\right) v_{0} \in N^{+}(R)$ (or equivalently $\left(1-\partial_{x}^{2}\right) v_{0} \in N^{-}(R)$ ), then there exists a constant $c>0$ independent of $\varepsilon$ and $t$ such that the solution of problem (25) satisfies

$$
\left\|v_{\varepsilon x}\right\|_{L^{\infty}} \leq c e^{c t}
$$

Proof. Using Lemmas 5 and 9, if $v_{0} \in H^{s}(R)$ with $1 \leq s \leq 3 / 2$, we have

$$
\left\|v_{\varepsilon}\right\|_{L^{\infty}(R)} \leq c\left\|v_{\varepsilon}\right\|_{H^{1}(R)} \leq c e^{c t}\left\|v_{\varepsilon 0}\right\|_{H^{1}(R)} \leq c e^{c t}
$$

where $c$ is independent of $\varepsilon$ and $t$.

From Lemma 8, we have

$$
\left\|v_{\varepsilon x}\right\|_{L^{\infty}(R)} \leq\left\|v_{\varepsilon}\right\|_{L^{\infty}(R)},
$$

which completes the proof.

\section{Proof of Main Results}

Proof of Theorem 1. Since $\|v\|_{L^{\infty}(R)} \leq c\|v\|_{H^{1}(R)} \leq c e^{c t}$ and taking $q+1=s$ in inequality (10), we have

$$
\|v\|_{H^{s}}^{2} \leq\left\|v_{0}\right\|_{H^{s}}^{2}+c \int_{0}^{t} e^{c \tau}\|v\|_{H^{s}}^{2}\left(\left\|v_{x}\right\|_{L^{\infty}}+\left\|v_{x}\right\|_{L^{\infty}}^{2}\right) d \tau
$$

from which we obtain

$$
\|v\|_{H^{s}} \leq\left\|v_{0}\right\|_{H^{s}} e^{c \int_{0}^{t} e^{c \tau}\left(\left\|v_{x}\right\|_{L^{\infty}}+\left\|v_{x}\right\|_{L^{\infty}}^{2}\right) d \tau} .
$$

Applying Lemma 8 yields

$$
\|v\|_{H^{s}} \leq\left\|v_{0}\right\|_{H^{s}} c e^{e^{c t}}
$$

from which we complete the proof of Theorem 1 .
Provided that $1 \leq s \leq 3 / 2$, for problem (25), applying Lemmas 5, 8, and 10, and the Gronwall's inequality, we obtain the inequalities

$$
\begin{gathered}
\left\|v_{\varepsilon}\right\|_{H^{1}} \leq\left\|v_{\varepsilon 0}\right\|_{H^{1}} \leq c e^{c t}, \\
\left\|v_{\varepsilon}\right\|_{H^{q}} \leq c\left\|v_{\varepsilon 0}\right\|_{H^{q}} \exp \left[\int_{0}^{t}\left(\left\|v_{\varepsilon x}\right\|+\left\|v_{\varepsilon x}\right\|_{L^{\infty}}^{2}\right) d \tau\right] \leq c e^{e^{c t}}, \\
\left\|u_{\varepsilon t}\right\|_{H^{r}} \leq c\left\|u_{\varepsilon}\right\|_{H^{r+1}}\left(1+e^{c t}\right) \leq c\left(1+e^{c t}\right),
\end{gathered}
$$

where $q \in(0, s], r \in[0, s-1]$, and $c$ is a constant independent of $t$ and $\varepsilon$. Using the Aubin compactness theorem, we know that that there is a subsequence $\left\{v_{\varepsilon_{n}}\right\}$ of $\left\{v_{\varepsilon}\right\}$ such that $\left\{v_{\varepsilon_{n}}\right\}$ and their temporal derivatives $\left\{v_{\varepsilon_{n} t}\right\}$ converge weakly to a function $v(t, x)$ and its derivative $v_{t}$ in the space $L^{2}\left([0, T], H^{s}(R)\right)$ and $L^{2}\left([0, T], H^{s-1}(R)\right)$, respectively, where $T$ is an arbitrary fixed positive number. In addition, for any real number $M_{1}>$ $0,\left\{v_{\varepsilon_{n}}\right\}$ converges strongly to the function $v$ in the space $L^{2}\left([0, T], H^{q}\left(-M_{1}, M_{1}\right)\right)$ for $q \in(0, s]$ and $\left\{v_{\varepsilon_{n} t}\right\}$ converges strongly to $v_{t}$ in the space $L^{2}\left([0, T], H^{r}\left(-M_{1}, M_{1}\right)\right)$ for $r \in[0$, $s-1]$.

Proof of Theorem 3. For an arbitrary fixed $T>0$, using Lemma 10 , we know that $\left\{v_{\varepsilon_{n} x}\right\}\left(\varepsilon_{n} \rightarrow 0\right)$ is bounded in the space $L^{\infty}$. Therefore, we derive that the sequences $\left\{v_{\varepsilon_{n}}\right\},\left\{v_{\varepsilon_{n} x}\right\}$, $\left\{v_{\varepsilon_{n} x}^{2}\right\}$, and $\left\{v_{\varepsilon_{n} x}^{3}\right\}$ converge weakly to $v, v_{x}, v_{x}^{2}$, and $v_{x}^{3}$ in $L^{2}$ $\left([0, T], H^{r}\left(-R_{1}, R_{1}\right)\right)$ for any $r \in[0, s-1)$, separately. Applying the identity $v^{m}\left(v_{x}^{2}\right)_{x}=\left(v^{m} v_{x}^{2}\right)_{x}-\left(v^{m}\right)_{x} v_{x}^{2}$, we conclude that $v$ satisfies the equation

$$
\begin{aligned}
&-\int_{0}^{T} \int_{R} v\left(\varphi_{t}-\varphi_{x x t}\right) d x d t \\
&=\int_{0}^{T} \int_{R}\left[\left(\frac{m+3}{m+2} v^{m+2}+(m+1) v^{m} v_{x}^{2}\right) \varphi_{x}\right. \\
&-\frac{1}{m+2} v^{m+2} \varphi_{x x x}-\frac{1}{2} v^{m} v_{x}^{2} \varphi_{x} \\
&\left.-\frac{m}{2} v^{m-1} v_{x}^{3} \varphi+\lambda v\left(\varphi-\varphi_{x x}\right)\right] d x d t
\end{aligned}
$$

where $\varphi(t, x) \in C_{0}^{\infty}([0, T] \times R)$. We know that $Y=L^{1}([0, T] \times$ $R)$ is a separable Banach space and $\left\{v_{\varepsilon_{n} x}\right\}$ is a bounded sequence in the dual space $Y^{*}=L^{\infty}([0, T] \times R)$ of $Y$. Thus, there exists a subsequence of $\left\{v_{\varepsilon_{n} x}\right\}$, still denoted by $\left\{v_{\varepsilon_{n} x}\right\}$, weakly star convergent to a function $u$ in $L^{\infty}([0, T] \times R)$. Since $\left\{v_{\varepsilon_{n} x}\right\}$ weakly converges to $v_{x}$ in $L^{2}([0, T] \times R)$, it derives that $v_{x}=u$ almost everywhere. Therefore, we obtain $v_{x} \in$ $L^{\infty}([0, T] \times R)$. Since $T>0$ is an arbitrary number, we complete the proof of existence of global weak solutions to problem (2).

\section{Conflict of Interests}

The authors declare that there is no conflict of interests regarding the publication of this paper. 


\section{Acknowledgment}

This work is supported by the Fundamental Research Funds for the Central Universities (JBK120504).

\section{References}

[1] M. Wu, "On the study of local solutions for a generalized Camassa-Holm equation," Abstract and Applied Analysis, vol. 2012, Article ID 164876, 17 pages, 2012.

[2] R. Camassa and D. D. Holm, "An integrable shallow water equation with peaked solitons," Physical Review Letters, vol. 3, no. 11, pp. 1661-1664, 1993.

[3] V. Novikov, "Generalizations of the Camassa-Holm equation," Journal of Physics A: Mathematical and Theoretical, vol. 42, no. 34, Article ID 342002, 2009.

[4] Z. Xin and P. Zhang, "On the weak solutions to a shallow water equation," Communications on Pure and Applied Mathematics, vol. 53, no. 11, pp. 1411-1433, 2000.

[5] G. M. Coclite, H. Holden, and K. H. Karlsen, "Global weak solutions to a generalized hyperelastic-rod wave equation," SIAM Journal on Mathematical Analysis, vol. 37, no. 4, pp. 10441069, 2005.

[6] A. Constantin and J. Escher, "Wave breaking for nonlinear nonlocal shallow water equations," Acta Mathematica, vol. 181, no. 2, pp. 229-243, 1998.

[7] A. Bressan and A. Constantin, "Global conservative solutions of the Camassa-Holm equation," Archive for Rational Mechanics and Analysis, vol. 183, no. 1, pp. 215-239, 2007.

[8] A. Bressan and A. Constantin, "Global dissipative solutions of the Camassa-Holm equation," Analysis and Applications, vol. 5, no. 1, pp. 1-27, 2007.

[9] H. Holden and X. Raynaud, "Dissipative solutions for the Camassa-Holm equation," Discrete and Continuous Dynamical Systems A, vol. 24, no. 4, pp. 1047-1112, 2009.

[10] H. Holden and X. Raynaud, "Global conservative solutions of the Camassa-Holm equation-a lagrangian point of view," Communications in Partial Differential Equations, vol. 32, no. 10-12, pp. 1511-1549, 2007.

[11] K. Grayshan, "Peakon solutions of the Novikov equation and properties of the data-to-solution map," Journal of Mathematical Analysis and Applications, vol. 397, no. 2, pp. 515-521, 2013.

[12] A. A. Himonas and C. Holliman, "The Cauchy problem for the Novikov equation," Nonlinearity, vol. 25, no. 2, pp. 449-479, 2012.

[13] A. N. W. Hone, H. Lundmark, and J. Szmigielski, "Explicit multipeakon solutions of Novikov's cubically nonlinear integrable Camassa-Holm type equation," Dynamics of Partial Differential Equations, vol. 6, no. 3, pp. 253-289, 2009.

[14] M. Wu and Y. Zhong, "The local strong and weak solutions for a generalized Novikov equation," Abstract and Applied Analysis, vol. 2012, Article ID 158126, 14 pages, 2012.

[15] S. Lai and Y. Wu, "A model containing both the Camassa-Holm and Degasperis-Procesi equations," Journal of Mathematical Analysis and Applications, vol. 374, no. 2, pp. 458-469, 2011.

[16] N. Li, S. Lai, S. Li, and M. Wu, "The local and global existence of solutions for a generalized Camassa-Holm equation," Abstract and Applied Analysis, vol. 2012, Article ID 532369, 26 pages, 2012. 


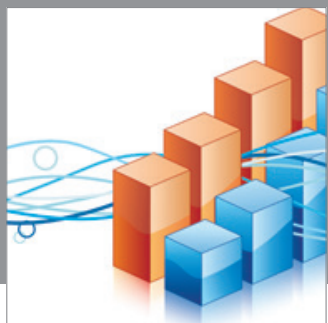

Advances in

Operations Research

mansans

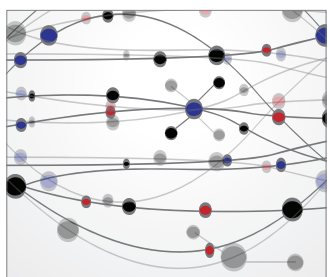

The Scientific World Journal
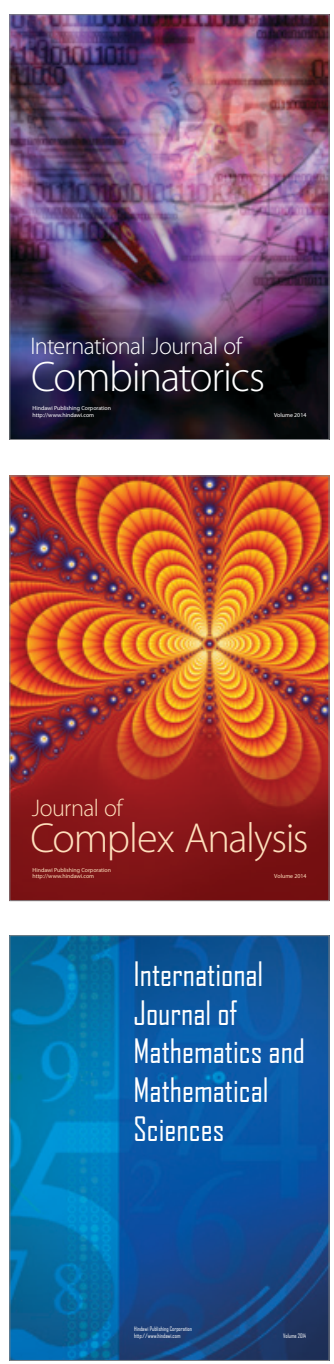
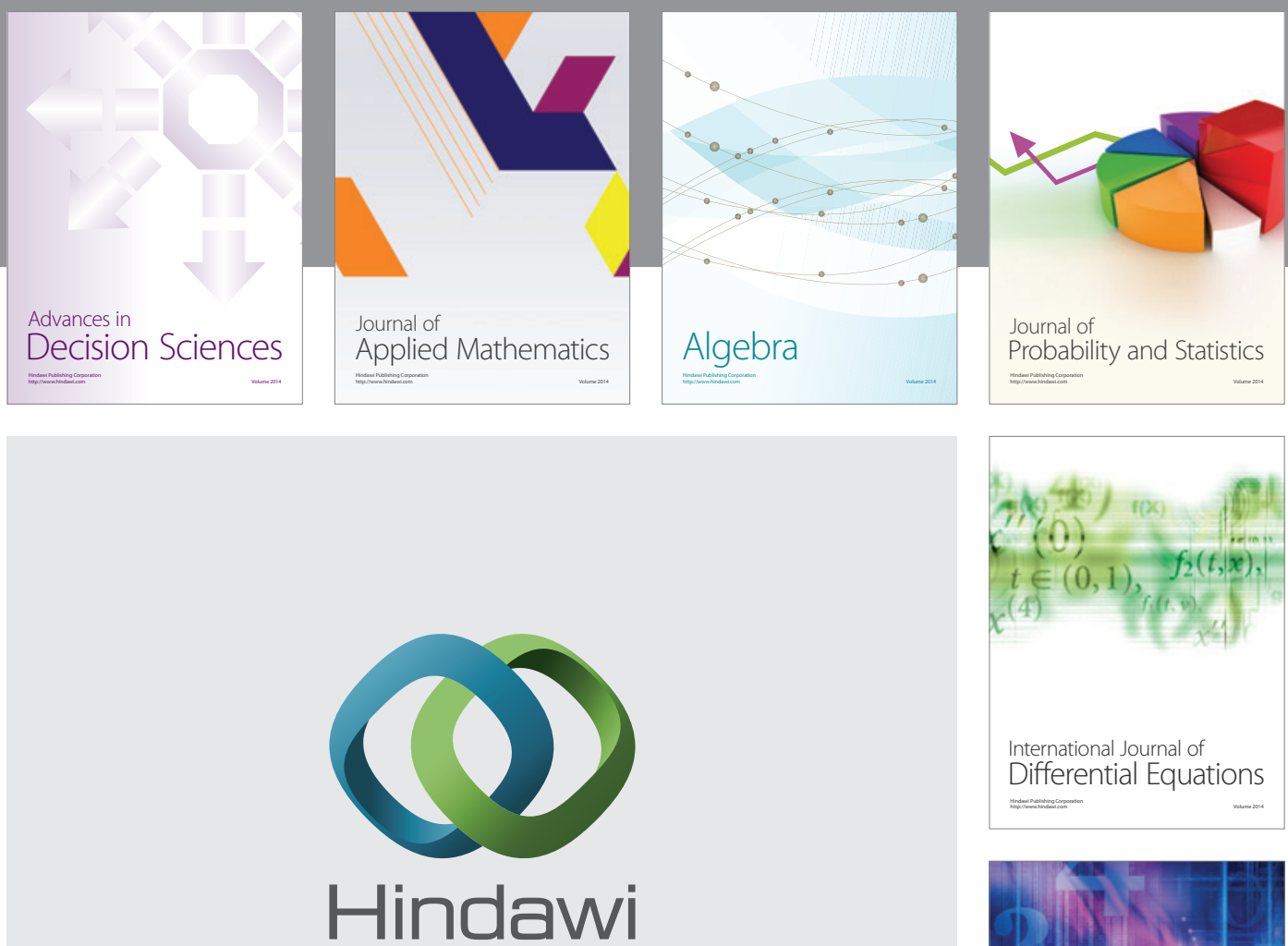

Submit your manuscripts at http://www.hindawi.com
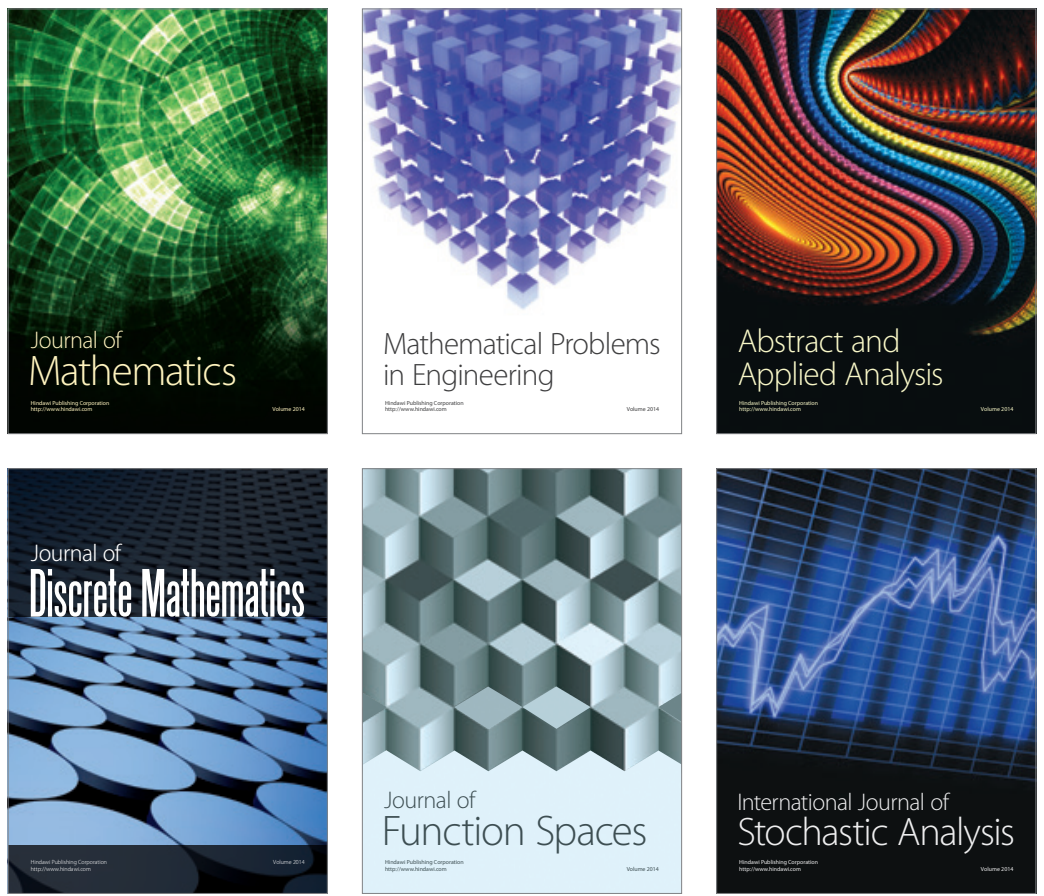

Journal of

Function Spaces

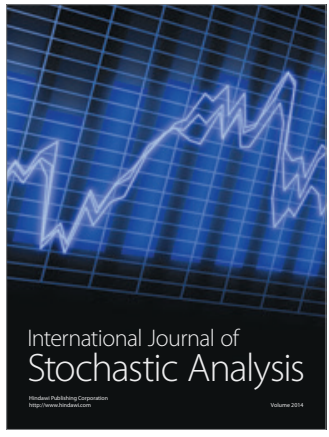

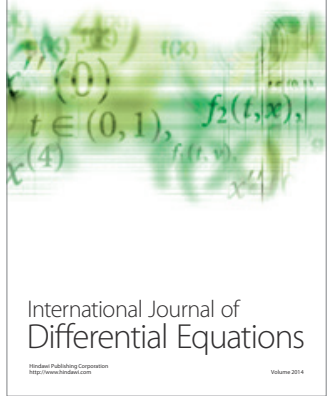
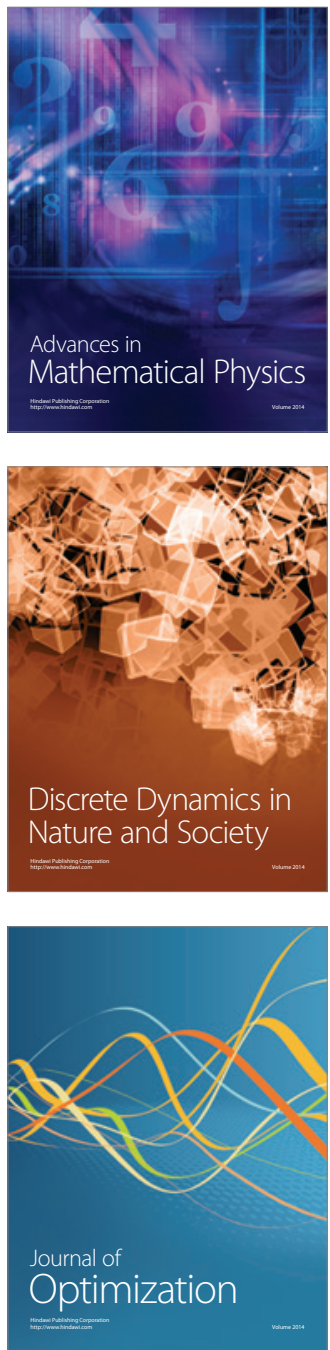